\title{
Estrategias de desarrollo rural con enfoque de género en Chile: el caso del programa "Formación y capacitación para mujeres campesinas"
}

Rural development strategies with gender perspective in Chile: the case of the program "Education and training for rural women"

Recibido: 15 de abril de 2015 - Revisado: 04 de octubre 2015 - Aceptado: 04 de diciembre de 2015

Sofía Boza Martínez ${ }^{2}$

Maruja Cortés Belmar ${ }^{3}$

Tomás Muñoz Eulogio

\section{Resumen}

La agricultura familiar campesina presenta limitaciones en sus capacidades productivas, las cuales se acentúan en el caso de las mujeres jefas de explotación, situación cada vez más frecuente en países de América Latina como Chile. En este contexto, el presente artículo tiene como objetivo analizar el caso específico del programa "Formación y capacitación para mujeres campesinas" del Instituto de Desarrollo Agropecuario de Chile, ejecutado en convenio con la Fundación para la Promoción y el Desarrollo de la Mujer. Lo anterior mediante la descripción de los principales elementos del programa, así como la caracterización de sus beneficiarias en un área específica.

\section{Palabras clave}

Desarrollo rural, programas públicos, género, agricultura familiar campesina, Chile.

\begin{abstract}
Family farming presents limitations in its production capacities, which are accentuated in the case of women heads of exploitation, an increasingly frequent situation in Latin America and Chile. In this context, this article aims to analyze the specific case of the program "Education and training for rural women" of the Instituto de Desarrollo Agropecuario de Chile, run in partnership with the Fundación para la Promoción y el Desarrollo de la Mujer. This is performed by describing the main elements of the program as well as the characterization of its beneficiaries in a specific area.
\end{abstract}

\section{Keywords}

Rural development, public policies, gender, family farming, Chile.

\footnotetext{
${ }^{1}$ Artículo de investigación elaborado en la Universidad de Chile.

2 Licenciada en Economía, máster en Desarrollo Económico y Políticas Públicas y doctora en Economía. Académica del Departamento de Economía Agraria de la Facultad de Ciencias Agronómicas y del Instituto de Estudios Internacionales de la Universidad de Chile, Santiago de Chile, Chile. Correo electrónico:

sofiaboza@u.uchile.cl

${ }^{3}$ Ingeniera agrónoma, máster en Desarrollo Rural y doctora en Economía. Académica del Departamento de Economía Agraria de la Facultad de Ciencias Agronómicas de la Universidad de Chile, Santiago de Chile, Chile.

Correo electrónico: marcortes@uchile.cl

${ }^{4}$ Ingeniero agrónomo. Asistente de proyectos del Departamento de Economía Agraria de la Facultad de Ciencias Agronómicas de la Universidad de Chile, Santiago de Chile, Chile. Correo electrónico: tomasfme@gmail.com

Para citar este artículo use: Boza, S., Cortés, M., \& Muñoz, T. (2016). Estrategias de desarrollo rural con enfoque de género en Chile: el caso del programa "Formación y capacitación para mujeres campesinas". Revista Civilizar Ciencias Sociales y Humanas, 16(30), 63-76.
} 


\section{Introducción}

En Chile, la agricultura familiar campesina (AFC) es responsable en gran medida de abastecer de alimentos al mercado nacional $\mathrm{y}$, por tanto, tiene un rol protagónico en la seguridad alimentaria del país. Sin embargo, este sector soporta importantes limitaciones que merman sus capacidades, sobre todo en el acceso a recursos productivos. Dicha situación se acentúa aún más cuando la jefatura predial está en manos de una mujer.

El sector público chileno ha destinado recursos al apoyo de la $\mathrm{AFC}$, sobre todo a través del accionar del Instituto de Desarrollo Agropecuario (Indap), una de las principales instituciones dedicadas al fomento productivo en Chile. Dentro de las iniciativas que desarrolla Indap, se encuentra el programa "Formación y capacitación para mujeres campesinas", ejecutado en convenio con la Fundación para la Promoción y el Desarrollo de la Mujer (Prodemu). La principal particularidad de este programa es que es el único en Chile dedicado de manera exclusiva a la atención de mujeres campesinas.

En este contexto, la presente investigación contempla dos preguntas centrales: a) cuáles son los principales elementos en los que se basa el programa "Formación y capacitación para mujeres campesinas", y b) cuáles son las características socioeconómicas de las beneficiarias del mismo en un área concreta del país. La respuesta a ambas cuestiones se espera contribuya a la orientación de posibles acciones de actualización del programa, o diseño en el futuro de otras iniciativas que contemplen beneficiarios similares. Para ello, el documento se estructura del siguiente modo: primero se presenta un marco teórico con base en los conceptos de AFC, género y desarrollo, luego se detalla la metodología que se empleó, para finalmente exponer los resultados obtenidos y las conclusiones.

\section{Marco conceptual: agricultura familiar campesina, género y desarrollo}

A pesar de tratarse de un término de uso común en la caracterización del sector agrícola, no existe una definición estandarizada de qué se entiende por AFC, dada la heterogeneidad de las condiciones en las que esta se despliega, lo que a su vez dificulta la concreción de las políticas dirigidas al sector (Salcedo, De la O, \& Guzmán, 2014). No obstante, Garner y De la O (2014) identificaron, tras realizar una revisión bibliográfica al respecto, algunos elementos especialmente recurrentes en la definición de la AFC, entre ellos: a) presencia de trabajo familiar, b) vinculación entre la administración predial y la jefatura del hogar, c) reducido tamaño predial y d) enfoque a la subsistencia.

Dada la estrecha relación de la AFC con las dinámicas familiares, resulta evidente que la evolución de los roles de género en los hogares rurales va a afectar la distribución de tareas y responsabilidades. En América Latina y el Caribe, las cifras censales muestran que el porcentaje de explotaciones agrícolas encabezadas por mujeres es del $16 \%$, lo que en algunos países, como Chile, estaría especialmente acentuado en la AFC (Organización de las Naciones Unidas para la Alimentación y la Agricultura -FAO, Comisión Económica para América Latina y el Caribe -CEPAL \& el Instituto Interamericano de Cooperación para la Agricultura - IICA, 2013; Qualitas, 2010). Lo anterior se condice con lo señalado por Foti (2009) para el caso de los Estados miembros del Mercado Común del Sur (Mercosur), donde la feminización de las tareas prediales incrementa cuanto menor es el tamaño de las explotaciones. Dichas tareas se darían para el caso de las mujeres en un espacio de estrecha coexistencia entre los roles productivo y reproductivo/doméstico, cuya conciliación deriva en jornadas prolongadas o en bajos rendimientos obtenidos mediante el primero de ellos.

Además, las mujeres campesinas en América Latina se enfrentan a restricciones en acceso 
a: tierras (Brumer, 2004; Deere \& León, 2001; 2003; Centro Latinoamericano para el Desarro1lo Rural- Rimisp, 2009; Zuluaga, 2011), recursos hídricos (Ruiz, 2012; 2013), financiamiento (Fletschner, 2009) y tecnología (Kleysen \& Campillo, 1996; Damián et al., 2008; FAO, 2011). Estas limitaciones derivan en que las explotaciones con jefatura femenina se aboquen a actividades poco rentables y, por ende, poco atractivas de expandir (World Bank, 2011).

A pesar de este diagnóstico, las políticas de desarrollo rural no se hicieron cargo por mucho tiempo de atender las inequidades de género en el ámbito productivo (Baca \& Herrera, 2008). De hecho, hasta los años setenta los programas de desarrollo circunscribían a la mujer al rol reproductivo/doméstico (Rico \& Gómez, 2009). A partir del declarado por Naciones Unidas como "Decenio de la mujer" (1975-1985), se producen importantes avances en la discusión respecto a la interacción de los asuntos de género en la planificación del desarrollo, la cual se plasmó en dos enfoques predominantes: mujer en el desarrollo (MED) y género y desarrollo (GED). El primero de ellos plantea la relevancia de mejorar la situación de las mujeres mediante su integración en las iniciativas de desarrollo, mientras que el segundo defiende la tarea de transformar las estructuras sociales que derivan en las inequidades hombre/mujer (Taylor, 1999).

Una de las principales críticas al enfoque GED es la dificultad de su puesta en práctica, lo que ha hecho que el enfoque MED haya tenido mayor trascendencia en las iniciativas de desarrollo (Rico \& Gómez, 2009); no solo en los países de menor renta, sino también en casos como el de la Unión Europea (Sabaté, 2009). Dichas iniciativas han priorizado la satisfacción de las categorizadas por Moser (1989) como necesidades de género "prácticas", frente a aquellas de carácter "estratégico". Lo precedente a pesar de que las condiciones estructurales que restringen el empoderamiento de la mujer constituyen un factor esencial que minora su capacidad empresarial $y$, en consecuencia, el impacto positivo de esta en el desarrollo rural (Buendía \& Carrasco, 2013).

En el caso de Chile, la política de desarrollo rural se ha caracterizado por apoyar el acceso a factores productivos en las explotaciones de menor tamaño, siendo la institución que canaliza mayor nivel de recursos en dicho sentido el Indap (Organización Internacional del Trabajo -OIT, \& Servicio de Cooperación Técnica -SERCOTEC, 2010; Sotomayor, Rodríguez, \& Rodrigues, 2011). Dentro de los programas del Indap, el único que atiende en exclusiva a mujeres es el de "Formación y capacitación para mujeres campesinas", ejecutado en convenio con la Fundación Prodemu, el cual es objeto de estudio en el presente artículo.

\section{Materiales y métodos}

Esta investigación es de carácter exploratorio-descriptivo, pues se pretende realizar una aproximación a una temática que ha tenido un cierto grado de tratamiento anterior, mediante la descripción de un caso específico. En concreto, se abordan las estrategias públicas de fomento del desarrollo rural orientadas a mujeres, a partir de la revisión de los principales elementos del programa "Formación y capacitación para mujeres campesinas" y la caracterización de un grupo de usuarias del mismo.

Para ello, se hace una contextualización general de los programas de fomento agrícola en Chile y se detallan los principales elementos que determinan al programa en estudio: propósito, público objetivo, institucionalidad, líneas de acción y evaluación. Todo ello con base en información secundaria.

Por último, se presentan los resultados de una encuesta que se efectuó entre los meses de junio y septiembre de 2013 a un total de 79 usuarias del programa, ubicadas en diez de las comunas de la Región Metropolitana de Santiago de Chile con una relativa mayor presencia de la actividad agrícola (Buín, Colina, Curacaví, 
Isla de Maipo, Lampa, El Monte, Paine, Pirque, San Pedro y Til Til). El cuestionario utilizado se conformó por preguntas relativas a: antecedentes generales de las encuestadas, características del territorio donde habitan, caracterización del grupo familiar y antecedentes laborales y productivos. Para el análisis de los datos se aplicó estadística descriptiva.

\section{Resultados}

\section{El fomento del desarrollo agrícola en Chile.}

Uno de los rasgos de la agricultura chilena es la "dualidad": una minoría de medianas y grandes empresas agrícolas enfocadas a la exportación y preponderante presencia de micro y pequeñas explotaciones familiares (Ríos \& Torres, 2014). En este sentido, según volumen de ventas, un 94,6\% de las explotaciones agrícolas del país podrían considerarse como microempresas, mientras que un 4,9\% serían pequeñas empresas (Aedo \& Alvear, 2010).

La alta presencia de micro y pequeñas empresas en el sector agrícola chileno podría ser vista a priori un obstáculo a la innovación. Aunque se han dado continuas mejoras en los últimos años, según la VIII Encuesta de Innovación del Ministerio de Economía, Fomento y Turismo para el periodo 2011-2012, solamente el $27,1 \%$ de las empresas identificadas como agrícolas celebró algún tipo de innovación, frente a un $49,6 \%$ en el sector energético, un 45 $\%$ en minería y un $33,8 \%$ en la industria manufacturera. Si dentro del sector se comparan las distintas empresas según su tamaño, efectivamente las pequeñas son aquellas con una menor tasa de innovación.

La disminución de esta brecha innovadora $\mathrm{y}$, en consecuencia, la mejora de la competitividad sectorial resulta clave para proteger el sector, y en especial a la pequeña agricultura, de shocks externos cada vez más recurrentes, como los eventos climáticos extremos (e.g. sequías y hela- das). En este sentido, la política agrícola en Chile se ha caracterizado por un acentuado fomento a la capacitación, asistencia técnica e inversión, muy por sobre a la entrega de ayudas directas no condicionadas (Sotomayor et al., 2011). Dentro de las entidades públicas chilenas dependientes del Ministerio de Agricultura ligadas al fomento productivo, aquella que concentra un mayor nivel de financiamiento desde inicios de los años noventa es Indap (OIT \& SERCOTEC, 2010). Según datos para 2013, el Indap atendía un total de 160.000 productores, de un universo potencial de 270.000 (Vial, 2014).

Según información actualizada al año 2013, el Indap ejerce su acción de apoyo a la AFC a través de una plataforma de 22 instrumentos, abordando tres áreas principales: desarrollo de capacidades; asistencia técnica y financiamiento de inversiones. Por otra parte, la población atendida puede ser clasificada como "multiactiva" y "comercial". En el primer caso, los usuarios complementan los ingresos que generan de la actividad predial con otras fuentes, mientras que en el segundo los rendimientos de la actividad agrícola les permiten dedicarse en exclusiva a ella (Martínez, Namdar-Irani, \& Sotomayor, 2014). Los usuarios multiactivos son beneficiarios de instrumentos como el Programa de Desarrollo Local, el Programa de Desarrollo Territorial Indígena, el Programa Agropecuario para el Desarrollo Integral y el Programa Indap-Prodemu; y los comerciales, de los servicios de asesoría técnica, el Programa de Gestión Empresarial y las alianzas productivas, entre otros.

Si bien es cierto, a todos los programas señalados las mujeres tienen por supuesto acceso, y en algunos de ellos su presencia ha sido predominante en años recientes (Boza, Cortés \& Guzmán, 2015), el Programa Indap-Prodemu es el único orientado exclusivamente a mujeres. Desde 2013 se atendió efectivamente a 3.668 usuarias, equivalente a un $2 \%$ del total de usuarios atendidos por Indap en dicho año (INDAP, 2014), por lo que se trata aún de un esfuerzo 
limitado. A continuación se amplía la descripción hasta ahora del programa, señalando sus principales aspectos.

\section{Programa "Formación y capacita- ción para mujeres campesinas".}

Desde su creación en el año 1993, el programa "Formación y capacitación para mujeres campesinas" ha atendido a más de 30000 beneficiarias provenientes de distintas regiones de Chile. Estas se caracterizan por pertenecer a familias rurales o estar ligadas a pequeñas productoras agrícolas, que se encuentran en situación de vulnerabilidad. Lo anterior, dentro del objetivo declarado de capacitarlas en la ejecución de iniciativas productivas agrícolas, estimulando el desarrollo de habilidades personales para emprendimiento y gestión, con el fin de que adquieran los conocimientos técnicos y las capacidades necesarias para generar negocios de manera competitiva y sustentable. Junto con esto, también se especifica como un factor relevante el lograr que las usuarias se desarrollen como personas integrales conscientes de sus derechos.

El programa es financiado a través de fondos de Indap y es ejecutado por Prodemu, cumpliendo por tanto estas dos entidades distintas funciones en relación con el mismo. Prodemu se encarga de la coordinación de todo el equipo de trabajo, la evaluación del programa y la retroalimentación con las usuarias, mientras Indap es responsable principalmente de brindar apoyo en los aspectos técnicos. El periodo de intervención tiene una duración total de tres años.

Acordecon ello se realiza una planificación mediante un plan de negocios trienal. Este es evaluado por un consultor externo y ajustado cada año por las usuarias acompañadas por un consejo directivo provincial y un equipo profesional de consultores. Además, dado que el programa contempla la inyección de fondos de inversión directa para la implementación de cada emprendimiento productivo, en un inicio se elabora un presupuesto de operación anual en coherencia con el plan de negocios trienal, que debe ser aprobado por el jefe de área de Indap y la directora provincial de Prodemu.

Por otra parte, con el objeto de fortalecer las competencias técnicas y personales de las usuarias, el programa procura que las mujeres completen su educación formal mínima derivándolas a entidades $a d h o c$, y ejecutando actividades de capacitación enfocadas en cuatro aspectos: gestión de negocios, fomento productivo de actividades silvoagropecuarias, artesanía y turismo, desarrollo organizacional y fomento de la asociatividad y desarrollo personal. En paralelo, el programa incluye jornadas intergrupales, un fondo de apoyo a la participación de dirigentes, giras técnicas dentro y fuera del país, eventos y jornadas públicas.

El desarrollo del programa es en el ámbito regional, implementándose por provincia, siendo el módulo su unidad operativa. Cada uno de estos módulos está compuesto por diez grupos, los cuales a su vez comprenden diez usuarias por grupo durante el primer año, un mínimo de siete en el segundo y cinco en el tercer año. El módulo está integrado por un grupo de mujeres con un nivel socioeconómico y formación técnica similares, que además comparten un mismo lugar geográfico que les permite desarrollar un proyecto común. Con el fin de propiciar la asociatividad en estos grupos, cada módulo cuenta con un coordinador campesino de área, profesional que además de guiar, fomenta la interacción de los grupos dentro de su módulo durante toda la intervención.

En el año 2012 se realizó una evaluación del impacto del programa en las beneficiarias egresadas en 2011. Para ello se encuestó a 336 mujeres en las regiones de Coquimbo, O’Higgins, Biobío y Metropolitana. Los resultados obtenidos muestran que el programa tuvo un impacto significativo en la tasa de ocupación y actividad de las beneficiarias, así como en consecuencia en su nivel de ingresos. Sin em- 
bargo, presenta dificultades en la capacidad de hacer perdurar los grupos de trabajo, más allá del mantenimiento de las actividades productivas individuales (Guernica Consultores, 2012).

\section{Caracterización de las mujeres beneficiarias del programa en Región Metropolitana}

\section{Antecedentes generales de las bene- ficiarias.}

Las mujeres encuestadas tienen edades que van de los 19 a los 81 años, con un promedio de 48 años. El $68 \%$ de las encuestadas se encontraba entre los 34 y los 62 años de edad. Así mismo, destaca el nivel educativo del grupo de mujeres, ya que un $35,4 \%$ declara haber terminado la educación media y esta cifra alcanza el $60,8 \%$ si se toman en cuenta a las usuarias que expresan haber al menos asistido a la educación superior. Estos datos son positivos si se considera que dentro de la población rural nacional, tan solo un 18,2 \% de las mujeres ha terminado la educación media (Qualitas, 2010).

Tabla 1

Nivel educacional de las mujeres encuestadas

\begin{tabular}{|lcc|}
\hline & Frecuencia & Proporción (\%) \\
\hline Básica completa & 10 & 12,7 \\
\hline Básica incompleta & 12 & 15,2 \\
\hline Media completa & 28 & 35,4 \\
\hline Media incompleta & 9 & 11,4 \\
\hline Técnica completa & 12 & 15,2 \\
\hline Técnica incompleta & 4 & 5,1 \\
\hline Profesional completa & 3 & 3,8 \\
\hline Profesional incompleta & 1 & 1,3 \\
\hline
\end{tabular}

Fuente: Elaboración propia.

En términos generales se podría afirmar que las mujeres encuestadas son un grupo relativamente bien educado, por lo que cuentan con mayores posibilidades de incorporarse al mundo laboral, ya sea de forma independiente o asalariada; como también debieran esperarse buenos resultados de las capacitaciones realizadas por el programa. No obstante, la relativamente elevada edad promedio es un factor que puede resultar limitante de las intenciones de modificar de manera significativa sus actividades productivas.

\section{Características del territorio.}

Desde el punto de vista del acceso a servicios, las mujeres encuestadas cuentan con una buena cobertura directa e indirecta en aquellos concernientes al área de la educación, ya que un $80 \%$ asevera disponer de jardines infantiles, un $87 \%$ de establecimientos de enseñanza básica y un $38 \%$ de establecimientos de enseñanza media en su sector. En cuanto al acceso a servicios del área de la salud, un 53 $\%$ de las encuestadas dice contar con posta de salud rural, un $42 \%$ con consultorio y un $20 \%$ con servicio de atención primaria de urgencia (Sapu).

Destaca en este ámbito el acceso de las usuarias a servicios relacionados con el cuidado de los niños pequeños y preadolescentes, como son los jardines infantiles y establecimientos de enseñanza básica respectivamente, ya que estas tareas revisten especial importancia debido a que en muchas ocasiones están vinculadas a actividades domésticas en que las mujeres utilizan una gran parte de su tiempo.

Tabla 2

Acceso a servicios en el sector donde viven

\begin{tabular}{|lcc|}
\hline & Frecuencia & Proporción (\%) \\
\hline Enseñanza parvularia & 63 & 79,7 \\
\hline Enseñanza básica & 69 & 87,3 \\
\hline Servicios de enseñanza media & 30 & 38,0 \\
\hline Consultorio público & 33 & 41,8 \\
\hline Sapu & 16 & 20,3 \\
\hline Posta de salud rural & 42 & 53,2
\end{tabular}

Fuente: Elaboración propia. 
Por tanto, el tener un acceso adecuado a los servicios mencionados incide en la carga de trabajo de las encuestadas, ya que está directamente relacionado con el rol reproductivo en lo que se ha venido a llamar como "doble jornada laboral" (Mauro, 2002).

Por otra parte, una situación característica de la Región Metropolitana es la cercanía que existe entre el medio urbano y el rural. En esta región se da un intenso flujo de circulación entre el campo y la ciudad, lo que queda de manifiesto al ser la región del país que presenta el mayor uso de sus caminos, además de contar con una extensa red pavimentada, concentrando junto con la $\mathrm{V}$ región un $19 \%$ de la red de caminos pavimentados nacionales (Figueroa \& Rozas, 2005). Lo dicho corresponde con que un $60,8 \%$ de las encuestadas declare movilizarse aprovechando algún servicio de transporte público y un $31,6 \%$ a través de automóvil particular. Tan solo un 7,6 \% de las mujeres declara movilizarse con medios de transporte no motorizados, como la bicicleta o a pie. Esta facilidad de acceso a núcleos urbanos puede resultar una ventaja a la hora de comercializar los productos generados y también en el aprovisionamiento de insumos.

\section{Situación de los hogares.}

El grupo familiar de las encuestadas está compuesto en promedio por cuatro personas, yendo desde grupos formados por once integrantes, hasta mujeres que viven solas. Dentro de los integrantes de los grupos, un $51 \%$ son mujeres y un $49 \%$ son hombres. Destaca en la composición de los hogares el gran número de adultos mayores, ya que representan un 17 $\%$ del total de los integrantes del grupo familiar. Esto condice con el progresivo envejecimiento que la población rural viene experimentando en las últimas décadas (Oxman, 2008).

Por otro lado, los menores de 18 años representan un $25 \%$, del cual un $9 \%$ corresponde a menores de 6 años de edad. Así mismo, llama la atención que la proporción de niños entre 7 y 18 años es mayor que la de niñas en el mismo rango, pero al pasar al siguiente, 19 a 64 años, la de hombres disminuye al compararla con la de mujeres. Lo anterior sugiere que los procesos migratorios, eminentemente masculinos, pueden haber afectado la composición de las familias de las mujeres encuestadas.

Tabla 3

Composición etaria de los hogares de las mujeres encuestadas

\begin{tabular}{|lccc|}
\hline & Mujeres (\%) & Hombres (\%) & Total (\%) \\
\hline 0-6 años & 2 & 7 & 9 \\
\hline $7-18$ años & 7 & 9 & 16 \\
\hline 19-64 años & 31 & 27 & 58 \\
\hline 765 años & 11 & 6 & 17 \\
\hline Total & 51 & 49 & 100 \\
\hline
\end{tabular}

Fuente: Elaboración propia.

Aunque mayoritariamente la estructura familiar tradicional (biparental) es la que prevalece-un $67,1 \%$ de las encuestadas declara vivir con su pareja- también son frecuentes los hogares monoparentales formados por la mujer, hijos y algún otro familiar, que se ven reflejados en el $32,9 \%$ de encuestadas que declara no vivir con su pareja.

Tabla 4

Situación de pareja de las mujeres encuestadas

\begin{tabular}{lcc} 
& Frecuencia & Proporción (\%) \\
\hline Soltera & 9 & 11,7 \\
\hline Con pareja & 18 & 23,4 \\
\hline Casada & 38 & 49,4 \\
\hline Separada & 2 & 2,6 \\
\hline Anulada & 1 & 1,3 \\
\hline Viuda & 9 & 11,7
\end{tabular}

Fuente: Elaboración propia. 
Es importante mencionar que si bien un alto porcentaje de los grupos familiares cuenta con ambos progenitores, en general es la mujer la que realiza la mayor parte de los quehaceres domésticos, con la consiguiente repercusión en la actividad laboral. Esto perpetúa los patrones tradicionales de organización de los grupos familiares en el campo, que limitan a la mujer a las labores domésticas y la sitúan en un plano de subordinación respecto al hombre (Valdés, 2007). Por otro lado, dentro de la situación de pareja de las encuestadas destaca el alto porcentaje de mujeres que se encuentran casadas o con pareja, ya que entre ambos alcanzan un $72,7 \%$ del total de los casos, con un $49,4 \%$ de mujeres que declararon estar casadas y un 23,4 $\%$ que declararon tener pareja.

Así mismo, a las encuestadas se les consultó sobre quién es la persona que toma las decisiones más importantes dentro del hogar. Dos datos sobresalen: a) la elevada cifra de mujeres encuestadas que declara que el proceso de toma de decisiones al interior del hogar se da de manera conjunta con su pareja, el cual llega a un $54,4 \%$, y b) el también significativo porcentaje de mujeres que afirma tomar las decisiones del hogar solas, lo que corresponde a un $27,8 \%$ de las encuestadas.

Tabla 5

Toma de decisiones en las familias de las mujeres encuestadas

\begin{tabular}{|lcc|}
\hline & Frecuencia & Proporción (\%) \\
\hline Usted & 22 & 27,8 \\
\hline Su pareja & 1 & 1,3 \\
\hline Entre usted y su pareja & 43 & 54,4 \\
\hline Su madre & 3 & 3,8 \\
\hline Su hija & 1 & 1,3 \\
\hline Su cuñada & 1 & 1,3 \\
\hline Su padre & 3 & 3,8 \\
\hline Usted y su hija & 1 & 1,3 \\
\hline $\begin{array}{l}\text { Entre todos los } \\
\text { miembros del hogar }\end{array}$ & 4 & 5,1 \\
\hline
\end{tabular}

Fuente: Elaboración propia.

\section{Situación productiva y laboral.}

Cuando a las encuestadas se les consultó sobre la situación laboral que mejor las representa, en su mayoría respondieron que "dueña de casa", con un 46,8 \%, seguido de "trabajadora" con un 13,9 \%. Esto llama la atención debido a que un gran número de las mujeres que se autodefinen como "dueñas de casa", pese a que desempeñan actividades laborales remuneradas fuera del hogar, no se reconocen a sí mismas como trabajadoras. Esto se puede interpretar desde la apreciación que las mujeres tienen de sí mismas y la valoración que dan al trabajo que realizan, ya que se desprende que la actividad remunerada es vista como accesoria. Esto es un reflejo de las dificultades sociales a las que se enfrentan las mujeres a la hora de incorporarse al mercado del trabajo, debido a que en gran medida la imagen de la "mujer trabajadora" aún está rodeada de prejuicios (Fawaz \& Soto, 2012).

Tabla 6

Situación laboral que mejor representa a las mujeres encuestadas

\begin{tabular}{|lcc|}
\hline & Frecuencia & Proporción (\%) \\
\hline Trabaja & 11 & 13,9 \\
\hline Trabaja y estudia & 5 & 6,3 \\
\hline Dueña de casa & 37 & 46,8 \\
\hline Jubilada o pensionista & 10 & 12,7 \\
\hline Cesante o busca trabajo & 4 & 5,1 \\
\hline $\begin{array}{l}\text { Trabaja } \\
\text { esporádicamente }\end{array}$ & 12 & 15,2 \\
\hline
\end{tabular}

Fuente: Elaboración propia.

En este contexto, para conocer cómo las mujeres encuestadas organizan su tiempo, se consultó en qué actividad utilizan la mayor parte de su jornada, en primera y segunda opción. Un $58,2 \%$ de las mujeres encuestadas declara como primera actividad los quehaceres del hogar, seguida del trabajo en la unidad productiva con un $17,7 \%$, siempre como primera opción. Como segunda alternativa, las mujeres declararon el 
trabajo en la unidad productiva con un 53,2 $\%$ seguido de los quehaceres del hogar con un $22,1 \%$. De este modo, los quehaceres del hogar y el trabajo en la unidad productiva son las dos principales actividades de las mujeres encuestadas, tanto en primera como segunda opción. Así se da cuenta de la gran carga que significa para las mujeres el hecho de asumir un trabajo remunerado fuera del hogar, ya que en la mayoría de los casos, eso no significa dejar de realizar las labores domésticas. A su vez, se confirma que en la mayor parte de los casos la actividad predial es vista como secundaria, lo que repercute en los rendimientos que de ella derivan y en su posible expansión en el futuro.

Lo precedente se refleja (entre otros factores) en los bajos ingresos de las mujeres encuestadas, los que en promedio alcanzan los CLP 74.934 mensuales (120 USD). Dicha cifra corresponde a tan solo un $36 \%$ del sueldo mínimo del país, el cual se fijó en CLP 210.000 en agosto del 2013; y se encuentra muy por debajo del ingreso promedio nacional de las mujeres, que alcanza los CLP 381.522 (García \& Larenas, 2011). Cabe mencionar que estos ingresos provienen principalmente del trabajo realizado en la actividad productiva ligada al programa y corresponden a un $23 \%$ de los ingresos totales del hogar, los que llegan a CLP 329.873 (529 USD) mensuales promedio.

Tabla 7

Principales rubros de los grupos de las mujeres encuestadas

\begin{tabular}{|lcc|} 
& Frecuencia & Proporción (\%) \\
\hline Apicultura & 5 & 6,3 \\
\hline Avicultura & 25 & 31,6 \\
\hline Floricultura & 9 & 11,4 \\
\hline Horticultura & 36 & 45,6 \\
\hline Helicicultura & 4 & 5,1 \\
\hline
\end{tabular}

Fuente: Elaboración propia.

Por último, desde el punto de vista productivo, los principales rubros a los que se de- dican las usuarias encuestadas son la horticultura y la avicultura, con un $45,6 \%$ y un $31,6 \%$ respectivamente, seguido de la floricultura con un $11,4 \%$. Es importante también destacar que muy pocos grupos funcionan formalmente, lo que se refleja en que tan solo un $17,9 \%$ de las encuestadas afirma que su unidad productiva inició actividades en el Servicio de Impuestos Internos. Esto impacta en la escasa utilización de canales formales de comercialización, lo que se observa en que un $75,4 \%$ de las encuestadas declara que los productos elaborados por el grupo se venden de manera directa en el lugar de producción y en un $16,4 \%$ de los casos la forma de distribución es "puerta a puerta". Lo anterior repercute finalmente en los bajos ingresos que logran aportar las mujeres a sus hogares.

\section{Conclusiones}

Considerando los resultados presentados, podemos decir que en Chile si bien el apoyo a la pequeña agricultura está presente en numerosos programas públicos (más de veinte solo en el caso de Indap) en el contexto de la política sectorial, son exiguas las iniciativas que se enfocan exclusivamente en la mujer campesina. De hecho, dentro de los programas desarrollados por Indap, principal institución en fomento de la AFC en el país, tan solo uno cumple dicha premisa. Esto a pesar de que casi una quinta parte de las explotaciones nacionales están en manos de mujeres, situación que se acentúa en particular en el caso de la AFC, y de que estas se encuentran sometidas a mayores limitaciones en su acceso a recursos productivos (incluido el tiempo) que los hombres.

Por otra parte, los resultados obtenidos de la encuesta realizada en la Región Metropolitana, muestran que estas usuarias del programa "Formación y capacitación para mujeres campesinas" evidencian condiciones propicias para el aprovechamiento del mismo, como son: su alto nivel educativo y buen acceso a servicios y a medios de transporte. Sin embargo, otros factores se sugieren como limitantes a las posibi- 
lidades de generar negocios más rentables, por ejemplo, el alto nivel de edad promedio, pero sobre todo la visión del trabajo predial como una actividad secundaria.

Además, o posiblemente relacionado de manera más o menos directa con lo anterior, el negocio se enmarca en un clima de "informalidad", con un muy bajo nivel de iniciación de actividades, así como de uso de canales formales de comercialización. Por tanto se hace necesario que el programa Indap-Prodemu, también aborde temas relativos a la comercialización y a la iniciación legal de actividades, de forma de posibilitar que las iniciativas perduren en el tiempo.

Considerando las limitantes señaladas para las usuarias del programa en la Región Metropolitana, es esperable que incidan en la capacidad de generar ingresos mediante la actividad productiva. Esto se demuestra en que mensualmente en promedio esta reditúa a las productoras la tercera parte de un salario mínimo. Aunque con ello no se pretende sugerir que el programa no sea viable, pero sí que tomando como referencia los objetivos que Indap declara respecto al mismo, es posible que el impacto obtenido esté más vinculado con el desarrollo de capacidades personales y el empoderamiento que con la generación de negocios que sean competitivos y sustentables en el tiempo en un sentido más amplio del término. Por ende, se sugiere que al evaluar el programa se consideren variables relativas al mejoramiento de las condiciones de las mujeres, más acorde con lo recogido por el enfoque GED, en lo que tiene que ver con la actuación sobre la estructura de las relaciones de género, y no tanto se tenga una visión finalista.

Por último, señalar que este estudio presenta ciertas limitaciones respecto a las encuestas efectuadas, ya que fueron tomadas solamente a productoras de la Región Metropolitana, a pesar de que el programa en análisis tiene cobertura nacional.

\section{Referencias}

Aedo, M., \& Alvear, J. J. (2010). Micro y Pequeña Empresa Agropecuaria en Chile: Criterios para una Focalización Eficiente de las Politicas para el Sector de acuerdo al VII Censo Agropecuario. Santiago, Chile: Indap.

Baca, N., \& Herrera, F. (2008). Emergencia de la relación desarrollo rural-género. Convergencia, Revista de Ciencias Sociales, 15(48), 223-253.

Boza, S., Cortés, M. \& Guzmán, F. (2015). Caracterización de pequeños empresarios agrícolas beneficiarios de programas de desarrollo local en la Región Metropolitana, Chile. IDESIA, 33(1), 135-142.

Brumer, A. (2004). Gênero e agricultura: a situação da mulher na agricultura do Rio Grande do Sul. Revista Estudos Feministas, 12(1), 205-227.

Buendía, I., \& Carrasco, I. (2013). Mujer, actividad emprendedora y desarrollo rural en América Latina y el Caribe. Cuadernos de desarrollo rural, 10(72), 21-45.

Centro Latinoamericano para el Desarrollo Rural- [RIMISP]. (2009). Acceso a la tierra en la agricultura familiar campesina como factor de desarrollo. Santiago, Chile: Oficina de Estudios y Políticas Agrarias. Recuperado de http://www.odepa.cl/wpcontent/files_mf/1369760654Tierra_en_ agricultura_familiar_campesina.pdf

Comisión Económica para América Latina y el Caribe -[CEPAL]., Instituto Interamericano de Cooperación para la Agricultura -[IICA]., \& Organización de las Naciones Unidas para la Alimentación y la Agricultura -[FAO]. (2013). Perspectivas de la agricultura y del desarrollo rural en las Américas: una mirada hacia 
América Latina y el Caribe 2014. San José, C. R.: IICA.

Damián, M. A., López, J. F., Ramírez, B., Parra, F., Paredes, J.A., Gil, A., \& Cruz,A. (2008). Hombres y mujeres en la producción de maíz: un estudio comparativo en Tlaxcala. Región y Sociedad, 20(42), 63-94.

Deere, C. D., \& León, M. (2001). Género, propiedad y empoderamiento: tierra, Estado y mercado en América Latina. Bogotá, Colombia: Tercer Mundo Editores; Universidad Nacional, Facultad de Ciencias Humanas.

Deere, C. D., \& León, M. (2003). The Gender Asset Gap: Land in Latin America. World Development, 31(6), 925-947.

Fawaz, J., \& Soto, P. (2012). Mujer, trabajo $\mathrm{y}$ familia. Tensiones, rupturas $\mathrm{y}$ continuidades en sectores rurales de Chile central. Revista de Estudios de Género La Ventana, 4(35), 218-254.

Figueroa, O., \& Rozas, P. (2005). Conectividad, ámbitos de impacto $y$ desarrollo territorial: el caso de Chile. Comisión Económica para América Latina y el Caribe. Santiago, Chile: CEPAL.

Fletschner, D. (2009). Rural Women's Access to Credit: Market Imperfections and Intra-household Dynamics. World Development, 37(3), 618-631.

Foti, M. P. (2009). Mujeres en la Agricultura familiar del MERCOSUR. Organización $e$ incidencia politica. Buenos Aires, Argentina: IGTN-ActionAid. Recuperado de http://www10.iadb.org/intal/intalcdi/ PE/2012/10301.pdf

García, A., \& Larenas, E. (2011). Compendio de Estadísticas de Género, Publicaciones del Instituto Nacional de Estadísticas
Desagregadas por Sexo (2007-2009). Santiago, Chile: Instituto Nacional de Estadísticas (INE).

Garner, E., \& De la O, A. P. (2014). Identifying the "family farming": an informal discussion of the concepts and definitions (ESA-FAO, Working Paper No. 14-10). Roma: FAO. Recuperado de http://www. fao.org/3/a-i4306e.pdf

Guernica Consultores (2012). Evaluación de resultados de beneficiarias del programa "Formación y Capacitación de Mujeres Campesinas" (INDAP-PRO$D E M U)$. Santiago, Chile. Recuperado de http://www.prodemu.cl/wp-content/ uploads/2015/06/2011_Informe-final_Evaluacion-Programa-Mujeres-Campesinas.pdf

Instituto de Desarrollo Agropecuario de Chile -[INDAP]. (2014). Lineamientos estratégicos 2014-2018. Por un Chile Rural Inclusivo. Santiago, Chile: INDAP.

Kleysen, B., \& Campillo, F. (1996). Productoras de alimentos en 18 países de América Latina y el Caribe: sintesis hemisférica. San José, Costa Rica: BID; IICA.

Lucas, R. (1988). On the Mechanics of Development Planning. Journal of Monetary Economics, 22(1), 3-42.

Martínez, H., Namdar-Irani, M., \& Sotomayor, O. (2014). Chile: más de 50 años de apoyo a la agricultura familiar campesina. En E. Sabourin, M. Samper \& O. Sotomayor, (Coords.), Politicas públicas $y$ agriculturas familiares en América Latina y el Caribe: Balance, desafios y perspectivas (pp. 103-124). Santiago, Chile: CEPAL

Mauro, A. 2002. La situación de la Mujer y las Brechas de Género: Análisis de la 
Información de la Encuesta CASEN 19902000. Santiago, Chile: MIDEPLAN.

Moser, C. (1989). Gender planning in the Third World: meeting practical and strategical gender needs. World Development. 17(11), 1799-1825.

Organización de las Naciones Unidas para la Alimentación y la Agricultura-[FAO]. (2011). The state of food and agriculture. Women in agreculture: Closing the gender gap for development. Roma, Italia: Autor.

Organización Internacional del Trabajo -[OIT]., \& Servicio de Cooperación Técnica [SERCOTEC]. (2010). La situación de la micro y pequeña empresa en Chile 2010. Santiago, Chile: OIT.

Oxman, V. (2008). Composición de los Hogares Rurales Chilenos en la Modernización Agropecuaria IN: La Mujer en la Agricultura Chilena, Resultados Censo Agropecuario 2007. Santiago, Chile: Instituto Nacional de Estadísticas.

Qualitas (2010). Estudio de caracterización de la pequeña agricultura a partir del VII Censo NacionalAgropecuarioy Forestal.Santiago, Chile: Qualitas; Indap. Recuperado de http://www.odepa.cl/wp-content/files $\mathrm{mf} / 1369699269$ Estudio_hogares_de_las_ explotaciones_silvoagropecuarias.pdf

Rico, M., \& Gómez, J. M. (2009). Análisis de los enfoques sobre género y desarrollo: algunas reflexiones acerca de la mujer y su incorporación al proceso de desarrollo rural. En J. A. Orejas, O. Retortillo \& A. J. Piñeyroa (Coords.), Mujer, empresa y medio rural: $V$ Congreso Internacional Mercado de Trabajo y Relaciones Laborales (pp. 239-274). Palencia, España: Diputación de Palencia.

Ríos, S., \& Torres, G. (2014). El sector agropecuario en la región de Los Lagos y el paradigma
"Chile potencia alimentaria": desafíos para la política agraria nacional. Mundo Agrario, 15(29). Recuperado de http://www.redalyc. org/articulo.oa? $\mathrm{id}=84531879008$

Ruiz, L. E. (2012). Relaciones de género y mercados de derechos de agua y tierra en Chiapas. Región y Sociedad, 24(53), 55-89.

Ruiz, L. E. (2013). Segregación de género en la asignación de derechos al agua en los sistemas de riego campesino en Chiapas, México. Cuadernos de Desarrollo Rural, 10(72), 201-222.

Sabaté, A. (2009). La dimensión de género en las políticas españolas y europeas de desarrollo rural. En: Unión de Pequeños Agricultores (UPA) (Ed.), Agricultura familiar en España (pp. 103-108). Madrid: UPA.

Salcedo, S., De la O, A. P. \& Guzmán, L. (2014). El concepto de agricultura familiar en América Latina y el Caribe. En S. Salcedo \& L. Guzmán (Eds.), Agricultura Familiar en América Latina y el Caribe: Recomendaciones de política (pp. 17-33). Santiago, Chile: FAO.

Sotomayor, O., Rodríguez, A., \& Rodrigues, M. (2011). Competitividad, sostenibilidad e inclusión social en la agricultura. Nuevas direcciones en el diseño de políticas en América Latina y el Caribe. Santiago, Chile: CEPAL.

Taylor, V. (1999). A Quick Guide to Gender Mainstreaming in Development Planning. Londres: Commonwealth Secretariat.

Valdés, X. (2007). Notas sobre la metamorfosis de la familia en Chile. Comisión Económica para América Latina y el Caribe (CEPAL). Santiago, Chile: LOM Ediciones.

Vial, R. (Julio, 2014). Estrategias públicas de apoyo a la pequeña agricultura. Presen- 
tación realizada en Seminario llevado a cabo en la Facultad de Ciencias Agronómicas de la Universidad de Chile, Santiago, Chile.

World Bank. (2011). Gender Equality and Development. Washington D. C.: The World Bank.

Zuluaga, G. P.(2011). ElAcceso a la TierraAsunto Clave para las Mujeres Campesinas en Antioquia, Colombia. Rev. Fac. Nal. Agr. Medellín, 64(1), 5949-5960. Recuperado de http://www.scielo.org.co/pdf/rfnam/ v64n1/a18v64n01.pdf 
